\title{
IDENTIFICATION OF EMISSION LINES IN A METEOR SPECTRUM OBTAINED ON AUGUST 2, 2011
}

\author{
A.M.Mozgova ${ }^{1}$, J.Borovicka $^{2}$, P.Spurny ${ }^{2}$, K.I.Churyumov ${ }^{1}$ \\ ${ }^{1}$ Taras Shevchenko National University, Astronomical Observatory, Kyiv, Ukraine \\ ${ }^{2}$ Astronomical Institute, Ondrejov Observatory, 25165 Ondrejov, Czech Republic \\ alenamozgova@ukr.net, jiri.borovicka@asu.cas.cz,klimchur@ukr.net
}

ABSTRACT. 125 emission lines were found in a meteor spectrum obtained on August 2, 2011. The identification of most of the lines is given. The following species were found in the spectrum: CrI, FeI, MgI, SiI, AlI, MnI, CaI, TiI, NaI, FeII, CaII, MgII, SiII, SrII.

Key words: meteors, spectra, line identification

\section{Introduction}

Meteors are phenomena in the Earth's atmosphere caused by particles of cosmic origin. Meteor is an unpredictable and short-term phenomenon. To capture it is not so easy, but it is harder to get a good meteor spectrum.

It was found that the average time interval between two penetrations of cosmic particles with masses $10^{-2} \mathrm{~g}$ in the atmosphere of our planet is $7.2 \times 10^{-3} \mathrm{~s}$ (Churyumov et al., 2012). It is about 140 particles per second. That is a constant interaction of meteoric matter with Earth's atmosphere. Due to this we are able to explore the structure, to determine physical parameters and study the chemical composition of interplanetary matter.

The main method of study of meteoroid composition is meteor spectroscopy.

Each meteor spectrum has a great scientific value. By processing it we can get information about the chemical composition of the cosmic body that invaded into the Earth's atmosphere and the nature of the glow of its substance.

The research of meteor spectra will allow to establish the genetic relationships of meteoroids with comets and asteroids.

The results of studies of meteoric phenomena can be also used as data about the physical and chemical processes in the upper Earth's atmosphere.
Meteor spectra provide information on the ablation process, hydrodynamics of meteors and on meteoroid masses (Borovicka, 1999).

In this paper we present the results of lines identification in a meteor spectrum.

\section{Observational data}

The meteor spectrum studied here was obtained on August 2, 2011 at 21:56:11 UT. The observations were made at the Ondrejov Observatory (Astronomical Institute of the Academy of Sciences of the Czech Republic).

The beginning of the phenomenon was detected above the point with coordinates: $\lambda_{\mathrm{E}}=15^{\circ}, 7699, \varphi_{\mathrm{N}}=49^{\circ}, 3814$, and the end coordinates: $\lambda_{\mathrm{E}}=15^{\circ}, 61661, \varphi_{\mathrm{N}}=49^{\circ}, 13797$.

The beginning and end heights of the meteor event were $\mathrm{h}=115,71 \mathrm{~km}$ and $\mathrm{h}=80,628 \mathrm{~km}$ respectively. The average velocity of the meteoric body in the Earth's atmosphere was $48 \mathrm{~km} / \mathrm{s}$. The meteor was sporadic with very eccentric orbit with perihelion at 0,969 AU and inclination of $79,7^{\circ}$.

The spectral record was obtained with a fixed camera Tessar $(1: 4,5 ; \mathrm{f}=360 \mathrm{~mm})$ equipped with 600 grooves $/ \mathrm{mm}$ diffraction grating.

The spectrum was recorded on FOMAPAN $20024 \times 18$ $\mathrm{cm}$ sheet film. The fireball image was divided into 5 segments due to the rotating shutter, which covered the camera 15,2 times per second (Fig.1).

There are places with high intensities of spectral lines in the image. Clearly visible are the lines of the first and the second spectral order. The spectral line of CaII barely visible also in the third order. The dispersion in the first order is $45 \AA / \mathrm{mm}$. The spectral region $3500-6400 \AA$ was covered. Meteor wake can be seen between the segments.

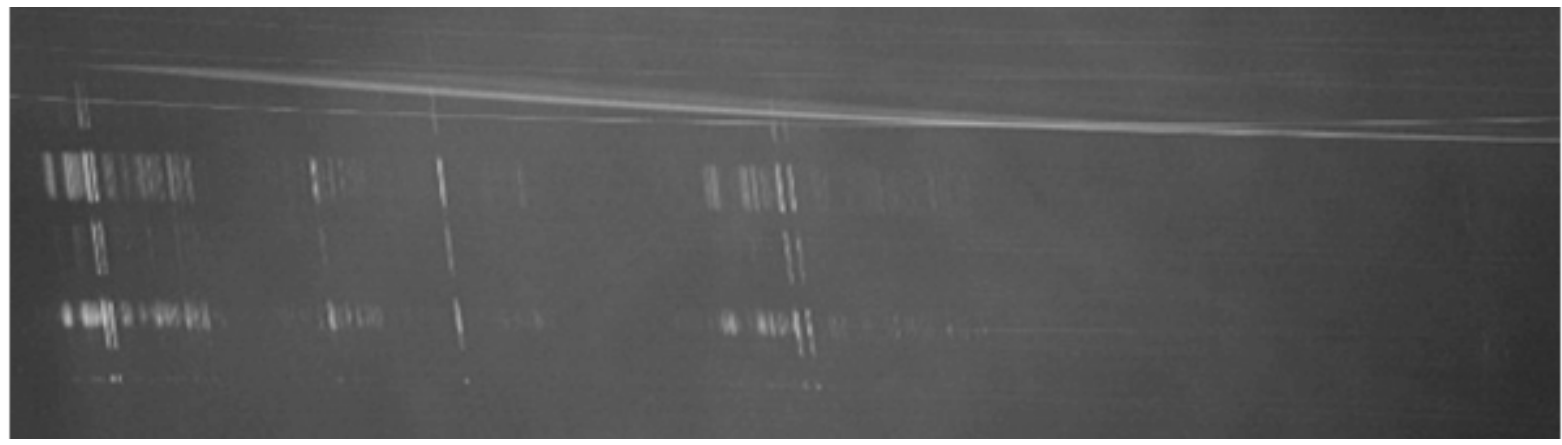

Figure 1: Meteor spectrum obtained on August 2, 2011. The flight direction was from the top to the bottom. The spectrum was divided into segments using rotating shutter. The first spectral order is on the left and a part of the second spectral order is in the middle. The horizontal trails are star zero order images. The bright star with spectrum is $\alpha$ Oph. 
Table 1. Identification of spectral lines

\begin{tabular}{|c|c|c|c|c|c|c|c|c|c|c|c|c|c|}
\hline no. & $\lambda_{\text {obs }}[\AA]$ & $\lambda[\AA]$ & Atom & $\begin{array}{c}\text { Measured } \\
\text { signal in } \\
\text { relative } \\
\text { units }\end{array}$ & $\begin{array}{l}\text { Intensities } \\
\text { in erg s-1 } \times \\
\times \AA^{-1} \operatorname{ster}^{-1}\end{array}$ & $|\Delta \lambda|$ & no. & $\lambda_{\text {obs }}[\AA]$ & $\lambda[\AA]$ & Atom & $\begin{array}{c}\text { Measured } \\
\text { signal in } \\
\text { relative } \\
\text { units }\end{array}$ & $\begin{array}{l}\text { Intensities } \\
\text { in erg s s-1 } \times \\
\times \AA^{-1} \operatorname{ster}^{-1}\end{array}$ & $|\Delta \lambda|$ \\
\hline \multirow{3}{*}{1} & 3685,50 & 3679,92 & FeI(5) & 97 & 355 & 5,58 & 54 & 4427,00 & 4427,31 & FeI(2) & 4166 & 1033 & 0,31 \\
\hline & & 3683,05 & \begin{tabular}{|l|}
$\mathrm{FeI}(5)$ \\
\end{tabular} & & & & 55 & 4435,00 & 4434,96 & $\mathrm{CaI}(4)$ & 112 & 34 & 0,04 \\
\hline & & 3687,46 & \begin{tabular}{|l}
$\mathrm{FeI}(21)$ \\
\end{tabular} & & & & 56 & 4455,00 & 4454,78 & $\mathrm{CaI}(4)$ & 102 & 33 & 0,22 \\
\hline \multirow{3}{*}{2} & 3708,00 & 3705,57 & \begin{tabular}{|l|} 
FeI $(5)$ \\
\end{tabular} & 164 & 528 & 2,43 & 57 & 4461,50 & 4461,65 & FeI(2) & 2356 & 593 & 0,15 \\
\hline & & 3707,83 & $\begin{array}{ll}\mathrm{FeI}(5) \\
\end{array}$ & & & & \multirow{3}{*}{58} & 4481,00 & 4481,24 & \begin{tabular}{|l}
$\operatorname{MgII}(4)$ \\
\end{tabular} & 5531 & 1369 & 0,24 \\
\hline & & 3709,25 & \begin{tabular}{|l|}
$\operatorname{FeI}(21)$ \\
\end{tabular} & & & & & & 4482,17 & \begin{tabular}{|l|}
$\mathrm{FeI}(2)$ \\
\end{tabular} & & & \\
\hline 3 & 3720,50 & 3719,94 & \begin{tabular}{|l|} 
FeI(5) \\
\end{tabular} & 784 & 2389 & 0,56 & & & 4482,26 & \begin{tabular}{|l} 
FeI(68) \\
\end{tabular} & & & \\
\hline 3 & & 3722,56 & FeI(5) & & & & 59 & 4489,50 & 4489,74 & \begin{tabular}{|l|}
$\mathrm{FeI}(2)$ \\
\end{tabular} & 235 & 59 & \\
\hline & & 3733,32 & \begin{tabular}{|l|}
$\mathrm{Fel}(5)$ \\
\end{tabular} & & & & 60 & 4493,50 & 4494,57 & \begin{tabular}{|l|}
$\mathrm{FeI}(68)$ \\
\end{tabular} & 109 & 27 & 1,07 \\
\hline 4 & & 3734,87 & \begin{tabular}{|l|l|} 
FeI(21) \\
\end{tabular} & & & & 61 & 4528,00 & 4528,62 & \begin{tabular}{|l} 
FeI(68) \\
\end{tabular} & 234 & 61 & 0,62 \\
\hline & 3736,50 & 3737,13 & $\begin{array}{ll}\mathrm{FeI}(5) \\
\end{array}$ & 1191 & 3391 & 0,63 & 62 & 4531,00 & 4531,15 & FeI(39) & 110 & 35 & 0,15 \\
\hline 5 & 3745,00 & 3745,56 & \begin{tabular}{|l|}
$\mathrm{FeI}(5)$ \\
\end{tabular} & 1804 & 4893 & 0,56 & 63 & 4549,00 & 4549,47 & \begin{tabular}{|l} 
FeII(38) \\
\end{tabular} & 99 & 29 & 0,47 \\
\hline 5 & & 3745,90 & FeI(5) & & & & & 4571,00 & 4571,10 & \begin{tabular}{|l|l}
$\operatorname{MgI}(1)$ \\
\end{tabular} & 264 & 73 & 0,10 \\
\hline & & 3763,79 & \begin{tabular}{|l|l|}
$\mathrm{FeI}(21)$ \\
\end{tabular} & & & & 64 & & 4571,98 & \begin{tabular}{|l|l|}
$\operatorname{TiII}(82)$ \\
\end{tabular} & & & \\
\hline 6 & 3766,50 & 3767,19 & $\begin{array}{l}\mathrm{FeI}(21) \\
\end{array}$ & 298 & 728 & 0,69 & 65 & 4583,00 & 4583,83 & \begin{tabular}{|l} 
FeII(38) \\
\end{tabular} & 117 & 37 & 0,83 \\
\hline & & 3798,51 & \begin{tabular}{|l|l}
$\mathrm{FeI}(21)$ \\
\end{tabular} & & & & 66 & 4647,00 & 4646,17 & \begin{tabular}{|l|}
$\operatorname{CrI}(21)$ \\
\end{tabular} & 53 & 17 & 0,20 \\
\hline 7 & 3798,00 & 3799,55 & $\begin{array}{ll}\mathrm{FeI}(21) \\
\end{array}$ & 263 & 556 & 1,55 & 66 & & 4647,43 & FeI(409) & & & \\
\hline 8 & 3815,00 & 3815,84 & FeI(45) & 314 & 577 & 0,84 & 67 & 4703,00 & 4702,99 & $\operatorname{MnI}(11)$ & 67 & 36 & 0,01 \\
\hline 9 & 3820,00 & 3820,43 & \begin{tabular}{|l} 
FeI $(20)$ \\
\end{tabular} & 701 & 1315 & 0,43 & 68 & 4859,50 & 4859,75 & $\mathrm{FeI}(318)$ & 51 & 37 & 0,25 \\
\hline 10 & 3824,50 & 3824,44 & \begin{tabular}{|l|}
$\mathrm{FeI}(4)$ \\
\end{tabular} & 1394 & 2562 & 0,06 & 69 & 4871,50 & 4871,32 & FeI(318) & 95 & 73 & 0,18 \\
\hline 10 & & 3825,88 & FeI $(20)$ & & & & & & 4872,14 & FeI(318) & & & \\
\hline 11 & & 3827,83 & \begin{tabular}{|l|l} 
FeI $(45)$ \\
\end{tabular} & & & & 70 & & 4890,76 & FeI(318) & & & \\
\hline 11 & 3828,50 & 3829,35 & $\operatorname{MgI}(3)$ & 1707 & 3061 & 0,85 & 70 & 4891,00 & 4891,50 & FeI(318) & 183 & 156 & 0,50 \\
\hline 12 & 3832,00 & 3832,30 & $\operatorname{Mgl}(3)$ & 2149 & 3767 & 0,30 & 71 & & 4919,00 & $\mathrm{FeI}(318)$ & & & \\
\hline 13 & 3837,50 & 3838,29 & $\operatorname{MgI}(3)$ & 2358 & 4018 & 0,79 & & 4920,00 & 4920,51 & FeI(318) & 234 & 230 & 0,51 \\
\hline 14 & 3856,00 & 3856,37 & \begin{tabular}{|l|}
$\mathrm{FeI}(4)$ \\
\end{tabular} & 1008 & 1549 & 0,37 & 72 & 4923,50 & 4923,92 & \begin{tabular}{|l|} 
FeII(42) \\
\end{tabular} & 244 & 243 & 0,42 \\
\hline 15 & 3859,50 & 3859,91 & FeI(4) & 3733 & 5566 & 0,41 & & & 4957,30 & $\mathrm{FeI}(318)$ & & & \\
\hline 16 & & 3878,02 & \begin{tabular}{|l} 
FeI $(20)$ \\
\end{tabular} & & & & 73 & 4957,50 & 4957,60 & FeI(318) & 354 & 414 & 0,10 \\
\hline 16 & 3878,00 & 3878,58 & \begin{tabular}{|l|}
$\mathrm{FeI}(4)$ \\
\end{tabular} & 963 & 1309 & 0,58 & & 4983,00 & 4982,81 & \begin{tabular}{|l|}
$\mathrm{NaI}(9)$ \\
\end{tabular} & 57 & 75 & 0,19 \\
\hline 17 & 3886,00 & 3886,28 & \begin{tabular}{|l|l|}
$\mathrm{FeI}(4)$ \\
\end{tabular} & 2403 & 3074 & 0,28 & 74 & & 4981,73 & \begin{tabular}{|l}
$\operatorname{Til}(38)$ \\
\end{tabular} & & & \\
\hline 18 & 3894,50 & 3895,66 & FeI(4) & 1161 & 1418 & 1,16 & 75 & 4994,00 & 4994,13 & FeI(16) & 44 & 68 & 0,13 \\
\hline 19 & 3899,00 & 3899,71 & FeI(4) & 935 & 1125 & 0,71 & 76 & 5006,00 & 5006,13 & FeI(318) & 49 & 90 & 0,13 \\
\hline & & 3902,95 & FeI(45) & & & & 77 & 5012,00 & 5012,07 & FeI(16) & 61 & 127 & 0,07 \\
\hline 20 & 3905,50 & 3905,53 & SiI(3) & 1179 & 1346 & 0,03 & 78 & 5018,50 & 5018,43 & FeII(42) & 294 & 428 & 0,07 \\
\hline & & 3906,48 & $\mathrm{FeI}(4)$ & & & & & 5041,50 & 5041,07 & \begin{tabular}{|l|}
$\mathrm{FeI}(16)$ \\
\end{tabular} & & & \\
\hline & & 3920,26 & FeI(4) & & & & 79 & & 5041,76 & \begin{tabular}{|l|}
$\mathrm{FeI}(36)$ \\
\end{tabular} & 173 & 267 & 0,26 \\
\hline 21 & 3922,50 & 3922,91 & $\mathrm{FeI}(4)$ & 1231 & 1250 & 0,41 & 80 & 5051,50 & 5051,64 & $\begin{array}{ll}\mathrm{FeI}(16) \\
\end{array}$ & 58 & 92 & 0,14 \\
\hline 22 & 3933,50 & 3933,67 & CaII(1) & 43294 & 41074 & 0,17 & 81 & 5056,00 & 5056,02 & \begin{tabular}{|l|}
$\operatorname{SilI}(5)$ \\
\end{tabular} & 86 & 133 & 0,02 \\
\hline 23 & 3961,50 & 3961,53 & AlI(1) & 340 & 272 & 0,03 & 82 & 5110,50 & 5110,41 & FeI(1) & 670 & 1188 & 0,09 \\
\hline & 3968,50 & 3968,47 & CaII(1) & 36620 & 28239 & 0,03 & & 5168,00 & 5167,32 & $\operatorname{MgI}(2)$ & 8369 & 17538 & 0,68 \\
\hline 24 & & 3969,26 & \begin{tabular}{|l|} 
FeI(43) \\
\end{tabular} & & & & 83 & & 5167,49 & FeI(37) & & & \\
\hline 25 & 4005,50 & 4005,25 & FeI(43) & 191 & 122 & 0,25 & 84 & & 5171,60 & FeI(36) & & & \\
\hline & & 4030,76 & $\operatorname{MnI}(2)$ & & & & 84 & 5172,50 & 5172,68 & $\operatorname{MgI}(2)$ & 7948 & 16834 & 0,18 \\
\hline 26 & & 4033,07 & MnI(2) & & & & 85 & 5183,50 & 5183,60 & $\operatorname{MgI}(2)$ & 8033 & 17488 & 0,10 \\
\hline & 4034,00 & 4034,49 & $\operatorname{MnI}(2)$ & 941 & 546 & 0,49 & 86 & 5195,00 & 5194,94 & $\begin{array}{l}\text { FeI(36) } \\
\end{array}$ & 204 & 443 & 0,06 \\
\hline 27 & 4045,50 & 4045,82 & FeI(43) & 1646 & 921 & 0,32 & & & 5204,52 & $\operatorname{CrI}(7)$ & & & \\
\hline 28 & 4063,50 & 4063,60 & FeI(43) & 921 & 488 & 0,10 & 87 & 5205,50 & 5206,04 & $\mathrm{CrI}(7)$ & 334 & 710 & 0,54 \\
\hline 29 & 4071,50 & 4071,74 & FeI(43) & 827 & 428 & 0,24 & 88 & 5208,00 & 5208,44 & $\operatorname{CrI}(7)$ & 359 & 758 & 0,44 \\
\hline 30 & 4077,00 & 4077,71 & \begin{tabular}{|l|l|} 
SrII(1) \\
\end{tabular} & 370 & 212 & 0,71 & & & 5215,19 & FeI(553) & & & \\
\hline 31 & 4131,50 & 4132,06 & \begin{tabular}{|l|} 
FeI(43) \\
\end{tabular} & 606 & 268 & 0,56 & 89 & 5216,50 & 5216,28 & \begin{tabular}{|l|}
$\mathrm{FeI}(36)$ \\
\end{tabular} & 149 & 303 & 0,22 \\
\hline 32 & 4143,50 & 4143,87 & FeI(43) & 753 & 326 & 0,37 & & & 5217,40 & FeI(553) & & & \\
\hline 33 & 4201,50 & 4202,03 & \begin{tabular}{|l|} 
FeI(42) \\
\end{tabular} & 721 & 271 & 0,53 & 90 & 5227,00 & 5227,19 & \begin{tabular}{|l|}
$\mathrm{FeI}(37)$ \\
\end{tabular} & 1287 & 2533 & 0,19 \\
\hline 34 & 4216,00 & 4216,19 & \begin{tabular}{|l|}
$\mathrm{FeI}(3)$ \\
\end{tabular} & 1157 & 418 & 0,19 & 91 & 5233,00 & 5232,95 & FeI(383) & 122 & 233 & 0,05 \\
\hline 35 & 4226,50 & 4226,73 & \begin{tabular}{|l}
$\mathrm{CaI}(2)$ \\
\end{tabular} & 3824 & 1354 & 0,23 & & 5270,00 & 5269,54 & FeI(15) & 6577 & 10405 & 0,46 \\
\hline 36 & 4251,00 & 4250,79 & \begin{tabular}{|l|l|}
$\mathrm{FeI}(42)$ \\
\end{tabular} & 492 & 166 & 0,21 & 92 & & 5270,36 & \begin{tabular}{|l}
$\mathrm{FeI}(37)$ \\
\end{tabular} & & & \\
\hline 36 & & 4250,13 & FeI $(152)$ & & & & & 5328,00 & 5328,04 & FeI(15) & 3345 & 4456 & 0,04 \\
\hline 37 & 4254,00 & 4254,35 & $\mathrm{CrI}(1)$ & 1137 & 375 & 0,35 & 93 & & 5328,53 & \begin{tabular}{|l}
$\mathrm{FeI}(37)$ \\
\end{tabular} & & & \\
\hline 38 & 4260,00 & 4260,48 & \begin{tabular}{|l} 
FeI $(152)$ \\
\end{tabular} & 554 & 180 & 0,48 & 04 & & 5339,94 & FeI(553) & & & \\
\hline 39 & & 4271,16 & FeI $(152)$ & & & & 94 & 5341,00 & 5341,03 & \begin{tabular}{|l|} 
FeI(37) \\
\end{tabular} & 161 & 206 & 0,03 \\
\hline & 4271,50 & 4271,76 & \begin{tabular}{|l|l} 
FeI $(42)$ \\
\end{tabular} & 2174 & 690 & 0,26 & 95 & 5371,50 & 5371,49 & \begin{tabular}{|l} 
FeI $(15)$ \\
\end{tabular} & 1440 & 1758 & 0,01 \\
\hline 40 & 4275,00 & 4274,80 & CrI(1) & 2006 & 555 & 0,2 & 96 & 5383,00 & 5383,37 & FeI(1146) & 25 & 65 & 0,37 \\
\hline 40 & & 4274,60 & $\mathrm{CrI}(1)$ & & & & 97 & 5397,50 & 5397,13 & FeI(15) & 715 & 814 & 0,37 \\
\hline 41 & 4282,00 & 4282,41 & FeI(71) & 133 & 47 & 0,41 & 98 & 5406,00 & 5405,78 & FeI(15) & 681 & 766 & 0,22 \\
\hline 42 & 4289,50 & 4289,72 & CrI(1) & 498 & 152 & 0,22 & 99 & 5415,00 & 5415,20 & FeI(1165) & 51 & 57 & 0,20 \\
\hline 43 & 4293,50 & 4294,13 & FeI(41) & 692 & 208 & 0,63 & 100 & 5424,00 & 5424,07 & $\mathrm{FeI}(1146)$ & 48 & 78 & 0,07 \\
\hline 44 & 4298,00 & 4299,24 & $\begin{array}{ll}\text { FeI }(152) \\
\end{array}$ & 225 & 66 & 1,24 & 101 & 5430,00 & 5429,70 & \begin{tabular}{|l}
$\mathrm{FeI}(15)$ \\
\end{tabular} & 741 & 786 & 0,30 \\
\hline 45 & 4302,00 & 4302,52 & \begin{tabular}{|l}
$\mathrm{CaI}(5)$ \\
\end{tabular} & 91 & 38 & 0,52 & 102 & 5434,50 & 5434,53 & \begin{tabular}{|l}
$\mathrm{FeI}(15)$ \\
\end{tabular} & 285 & 298 & 0,03 \\
\hline 46 & 4307,50 & 4307,91 & \begin{tabular}{|l|} 
FeI(42) \\
\end{tabular} & 1750 & 506 & 0,41 & 103 & 5447,00 & 5446,92 & \begin{tabular}{|l}
$\mathrm{FeI}(15)$ \\
\end{tabular} & 592 & 607 & 0,08 \\
\hline 47 & 4325,50 & 4325,77 & \begin{tabular}{|l|l} 
FeII \\
\end{tabular} & 1801 & 513 & 0,27 & 104 & 5456,00 & 5455,61 & \begin{tabular}{|l} 
FeI(15) \\
\end{tabular} & 356 & 350 & 0,39 \\
\hline & 4340,00 & 4339,45 & CrI(22) & 92 & 31 & 0,55 & 105 & 5506,00 & 5506,78 & \begin{tabular}{|l} 
FeI(15) \\
\end{tabular} & 26 & 33 & 0,78 \\
\hline 48 & & 4339,72 & \begin{tabular}{|l|l|}
$\mathrm{CrI}(22)$ \\
\end{tabular} & & & & 106 & 5528,50 & 5528,41 & \begin{tabular}{|l}
$\operatorname{MgI}(9)$ \\
\end{tabular} & 139 & 105 & 0,09 \\
\hline & & 4351,05 & $\operatorname{CrI}(22)$ & & & & & 5587,00 & 5586,76 & FeI(686) & 106 & 61 & 0,24 \\
\hline 49 & 4351,50 & 4351,77 & \begin{tabular}{|l|l}
$\mathrm{CrI}(22)$ \\
\end{tabular} & 325 & 87 & 0,27 & 107 & & 5588,76 & \begin{tabular}{|l|}
$\mathrm{CaI}(21)$ \\
\end{tabular} & & & \\
\hline & & 4351,91 & $\operatorname{MgI}(14)$ & & & & 108 & 5593,50 & 5594,47 & $\mathrm{CaI}(21)$ & 27 & 16 & 0,97 \\
\hline 50 & 4375,50 & 4375,93 & FeI(2) & 4774 & 1235 & 0,43 & 109 & 5616,00 & 5615,65 & \begin{tabular}{|l|l}
$\mathrm{FeI}(686)$ \\
\end{tabular} & 183 & 90 & 0,35 \\
\hline 51 & 4383,50 & 4383,55 & \begin{tabular}{|l}
$\mathrm{FeI}(41)$ \\
\end{tabular} & 4671 & 1218 & 0,05 & 110 & 5688,00 & 5688,21 & \begin{tabular}{|l|}
$\mathrm{NaI}(6)$ \\
\end{tabular} & 101 & 37 & 0,21 \\
\hline 52 & 4404,50 & 4404,75 & \begin{tabular}{|l|l|}
$\mathrm{FeI}(41)$ \\
\end{tabular} & 2015 & 511 & 0,25 & 111 & 5890,00 & 5889,95 & $\mathrm{NaI}(1)$ & 24016 & 11255 & 0,05 \\
\hline 53 & 4414,50 & 4415,13 & $\begin{array}{ll}\text { FeI }(41) \\
\end{array}$ & 769 & 190 & 0,63 & 112 & 5895,50 & 5895,92 & $\mathrm{NaI}(1)$ & 16425 & 8796 & 0,42 \\
\hline
\end{tabular}




\begin{tabular}{|c|c|c|c|c|c|c|c|c|c|c|c|c|c|}
\hline no. & $\lambda_{\text {obs }}[\AA]$ & $\lambda[\AA]$ & Atom & $\begin{array}{l}\text { Measured } \\
\text { signal in } \\
\text { relative } \\
\text { units }\end{array}$ & $\begin{array}{c}\text { Intensities } \\
\text { in } \operatorname{erg~s}^{-1} \times \\
\times \AA^{-1} \text { ster }^{-1}\end{array}$ & $|\Delta \lambda|$ & no. & $\lambda_{\text {obs }}[\AA]$ & $\lambda[\AA]$ & Atom & $\begin{array}{l}\text { Measured } \\
\text { signal in } \\
\text { relative } \\
\text { units }\end{array}$ & $\begin{array}{l}\text { Intensities } \\
\text { in erg s } \mathrm{s}^{-1} \times \\
\times \AA^{-1} \text { ster }^{-1}\end{array}$ & $|\Delta \lambda|$ \\
\hline 113 & 6101,50 & 6102,72 & $\mathrm{CaI}(3)$ & 53 & 23 & 1,22 & 119 & 6229,50 & 6230,73 & $\mathrm{FeI}(207)$ & 78 & 34 & 1,23 \\
\hline 114 & 6121,00 & 6122,22 & $\mathrm{CaI}(3)$ & 81 & 34 & 1,22 & 120 & 6245,50 & 6246,33 & $\mathrm{FeI}(816)$ & 42 & 20 & 0,83 \\
\hline \multirow{2}{*}{115} & 6136,00 & 6136,62 & FeI(169) & 87 & 37 & 0,62 & 121 & 6251,50 & 6252,56 & FeI(169) & 64 & 29 & 1,06 \\
\hline & & 6137,70 & FeI(207) & & & & 122 & 6334,00 & 6335,34 & $\mathrm{FeI}(62)$ & 5206 & 34 & 1,34 \\
\hline 116 & 6161,00 & 6162,17 & $\mathrm{CaI}(3)$ & 204 & 86 & 1,17 & 123 & 6346,50 & 6347,10 & SiII(2) & 472 & 354 & 0,60 \\
\hline \multirow{2}{*}{117} & & 6169,06 & $\mathrm{CaI}(20)$ & & & & 124 & 6358,00 & 6358,69 & FeI(13) & 49 & 37 & 0,69 \\
\hline & 6169,50 & 6169,56 & $\mathrm{CaI}(20)$ & 54 & 22 & 0,06 & 125 & 6370,50 & 6371,36 & SiII(2) & 142 & 131 & 0,86 \\
\hline 118 & 6190,50 & 6191,56 & FeI(169) & 55 & 24 & 1,06 & & & & & & & \\
\hline
\end{tabular}

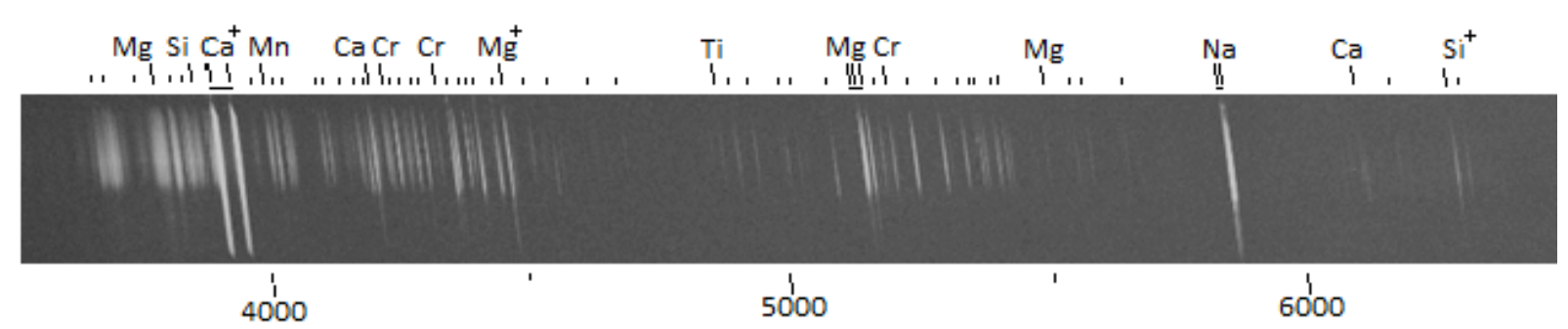

Figure 2: A part of the meteor spectrum during the flare. The important lines are identified. The short dashes show iron lines.

\section{Identification of spectral lines}

For spectral lines identification the brightest part of the first spectral order during the main flare which took place at the heights $h=83,5-84,5 \mathrm{~km}$ was used (Fig. 2). The spectrum was scanned and the spectrogram was obtained. The plate background was measured between segments. The measurements were calibrated. The wavelengths scale was determined by means of known lines in the spectrum (Borovicka, 1993). Polynomial fit of degree 3 was used to relate the instrumental lengths to wavelengths.

For photometric calibration the characteristic curve was constructed from the zero orders of stars recorded on the plate. Limiting magnitude was 4 . Figure 3 shows the linear part of the characteristic curve.

The relative spectral sensitivity of the system was determined by using the first order spectrum of Jupiter and Polar Star recorded on the same plate. The real energy distribution in the Jupiter's spectrum was taken from Karkoschka (1994). The resulting relative spectral sensitivity function is shown in figure 4 . The maximum of sensitivity is around $4500 \AA$ and minimum occurs at $5200 \AA$.

The spectrum includes many individual emission lines belonging to atoms of different chemical elements.

There are 125 emission lines identified in the meteor spectrum. For identification the tables of Moore (1945) and Borovicka (1994) were used.

The identification of observed spectral lines is given in Table 1. The following quantities are given in Table 1: Number and wavelength of the observed line, catalogue wavelength, identification (atom and multiplet), measured signal in relative units, intensities in $\operatorname{erg~} \mathrm{s}^{-1} \AA^{-1}$ ster $^{-1}$, difference between wavelength of the observed line and catalogue wavelength.

\section{Conclusion}

The basic processing of meteor spectrum was made. The meteor spectrogram was obtained and the identification of 125 emission lines found in the spectrum is presented. They were formed by CrI, FeI, MgI, SiI, AlI, MnI, CaI, TiI, NaI, FeII, CaII, MgII, SiII, SrII. Further analysis and theoretical interpretation of the spectrum is forthcoming.

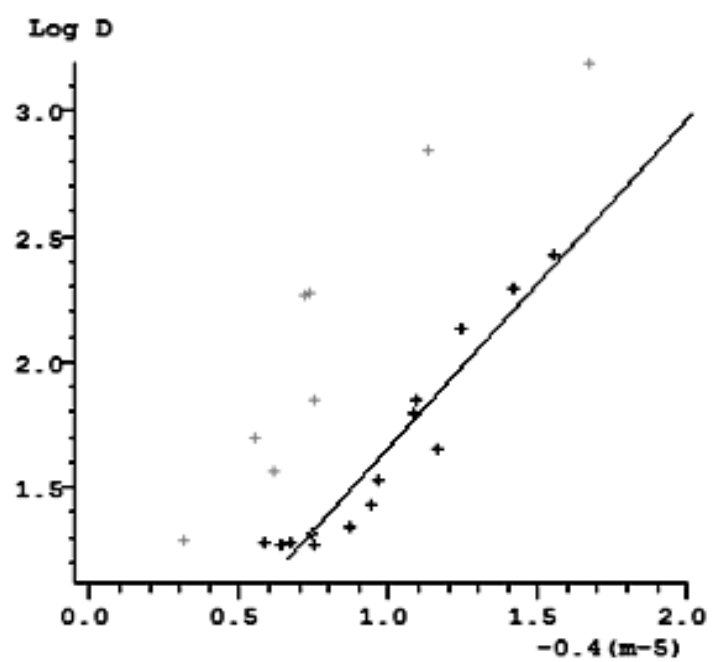

Figure 3: Characteristic curve of the plate. The opacity as a function of incident flux given by the stellar magnitude $\mathrm{m}$. The crosses represent individual stars. Grey crosses were omitted from the fit.

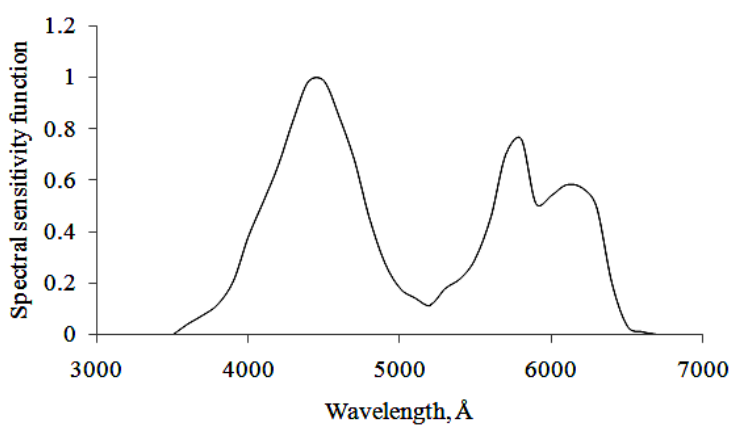

Figure 4: Relative spectral sensitivity of the spectrograph.

\section{Referenses}

Borovicka J.: 1993, Astron. Astrophys., 279, 627.

Borovicka J.: 1994, Astron. Astrophys. Suppl. Ser., 103, 83.

Borovicka J.: 1999, in: METEOROIDS 1998, Astron. Inst., Slovak Acad. Sci., Bratislava, 335.

Churyumov K. et al.: 2012, Physics and Astronomy in modern school, 1, N 16, 51.

Karkoscka E.: 1994, Icarus, 111, 174.

Moore Ch.E.: 1945, A Multiplet Table of Astrophys. Interest, Contrib. Princeton Univ. Obs., 20. 